\title{
Relación entre las características periodontales y la sonrisa gingival en niños: un estudio de casos y controles
}

\author{
Lucía V. Bernal, Esp. , Óscar Zapata N., Esp. , Diana María Barbosa-Liz ${ }_{1}^{*}$ \\ $\mathrm{MsC}_{{ }_{1}}$, Flavio Estrada, Esp. ${ }_{1}$, Laura Ángel, Esp. ${ }_{1}$
}

${ }_{1}$ Universidad de Antioquia, Facultad de Odontología, Medellín, Colombia

Recibido: 1 de octubre del 2015 Aprobado: 9 de noviembre del 2015

*Autor de correspondencia: Diana María Barbosa-Liz. Universidad de Antioquia, Facultad de Odontología. Calle 70 n. ${ }^{52-21,}$ Medellín, Colombia. Teléfono: (57-4) 2196772. Correo electrónico: diana.barbosa@udea.edu.co

Cómo citar este artículo: Bernal LV, Zapata Ó, Barbosa DM, Estrada F, Ángel L. Relación entre las características periodontales y la sonrisa gingival en niños: un estudio de casos y controles. Rev Nac Odontol. 2016;12(22):7-16. doi: http:// dx.doi.org/10.16925/od.v12i22.1201

Resumen. Introducción: la presencia de sonrisa gingival (SG) es uno de los aspectos más negativamente valorados. Su presencia en la vida adulta es un motivo de consulta constante, pero en la niñez tiende a ser considerado como normal. El objetivo de este estudio fue describir y comparar las características del periodonto de un grupo de niños, con diferentes maloclusiones, con y sin sonrisa gingival, y evaluar la influencia de las características gingivales sobre la presencia de sonrisa gingival. Métodos: en este estudio analítico de casos y controles, el tamaño muestral fue probabilístico. Se incluyeron 163 niños en dentición mixta silente que cumplieron los criterios de inclusión y exclusión: 37 casos con sonrisa gingival (sG) y 126 controles sin sonrisa gingival (NSG). Se les evaluaron las variables oclusales, de sonrisa y periodontales por medio de un examen clínico y de un fotograma. Los evaluadores estaban previamente calibrados (kappa 0,87 e ICC 0,96). Resultados: la edad promedio de los pacientes en ambos grupos fue de 8,8 años con una desviación estándar de 0,9 para los casos y de 0,8 para los controles. La maloclusión más predominante fue la clase i $(63,8 \%$ de la muestra). Las características periodontales fueron similares en ambos grupos. El biotipo periodontal grueso tuvo una tendencia a comportarse como factor de riesgo para SG (OR 1,6) pero esta relación no fue estadísticamente significativa (IC $=95 \% 0,6-4,1)$. Conclusiones: los factores periodontales no influyen sobre la presencia de sonrisa gingival en niños; su comportamiento en etapas posteriores deberá ser observado.

Palabras clave: maloclusión, periodonto, sonrisa, tejidos blandos. 


\title{
Relation between Periodontal Characteristics and Gummy Smile in Children: A Case-Control Study
}

\begin{abstract}
Introduction: the gummy smile (Gs) is one of the most negatively valued aspects; its presence in the adult life is reason of constant consultation, but during childhood it usually is considered normal. The aim of this study was to describe and compare the periodontium traits in a group of children with different malocclusions with or without gummy smile, and evaluate the influence of gingival characteristics along with gummy smile. Methods: in this analytical study of case and control, the sample size was probabilistic. 163 children with mixed silent dentition that meted the criteria of inclusion and exclusion were involved: 37 cases with gummy smile (Gs) and 126 controls without gummy smile (NGs). The occlusal variables of smile and periodontal disease were assessed through a clinical exam and photograms. The evaluators were previously calibrated (kappa 0,87 and ICC $0,96)$. Results: the average age of patients in both groups was of 8,8 years with a standard deviation of 0,9 in cases and 0,8 in controls. The prevailing malocclusion was class I ( $63,8 \%$ of the samples). The periodontal characteristics were similar in both groups. The thick periodontal biotype had a tendency to behave as risk factor to Gs $($ or 1,6$)$ but the relation wasn't statistically significant (IC $=95 \% 0,6-4,1)$. Conclusion: the periodontal factors do not have an effect on gummy smile in children, though their behavior at later stages must be closely observed.
\end{abstract}

Keywords: malocclusion, periodontal, smile, soft tissue.

\section{Relação entre as características periodontais e o sorriso gengival em crianças: uma análise de casos e controles}

Resumo. Introdução: a presença de sorriso gengival (sG) é um dos aspectos com maior valoração negativa. A sua presença na vida adulta é um constante motivo de consulta, mas na infância geralmente é considerado normal. O escopo desde estudo foi descrever e comparar as características do periodonto de um grupo de crianças, com diferentes maloclusões, com e sem sorriso gengival, e avaliar a influência das características gengivais sobre a presença de sorriso gengival. Métodos: neste estudo analítico de casos e controles, o tamanho da amostra foi probabilístico. Foram incluídas 163 crianças em dentição mista silente que cumprissem os critérios de inclusão e exclusão: 37 casos com sorriso gengival (SG) e 126 controles sem sorriso gengival (NSG). Foram avaliadas suas variáveis oclusais, de sorriso e periodontais mediante exame clínico e de um fotograma. Os avaliadores estavam previamente calibrados (kappa 0,87 e ICC 0,96). Resultados: a idade média dos pacientes em ambos os grupos foi de 8,8 anos com uma desviacão padrão de 0,9 para os casos e de 0,8 para os controles. A maloclusão com maior predomínio foi a classe i ( $63,8 \%$ da amostra). As características periodontais foram similares em ambos os grupos. O biótipo periodontal grosso teve uma tendência a ser um fator de risco para SG (OR 1,6), mas essa maloclusão não foi estatisticamente significativa (IC $=95 \% 0,6-4,1)$. Conclusões: os fatores periodontais não incidem na presença de sorriso gengival em crianças; seu comportamento em estágios posteriores deverá ser observado.

Palavras-chave: maloclusão, periodonto, sorriso, tecidos brandos. 


\section{Introducción}

El periodonto está constituido por las estructuras que se localizan alrededor del diente; su función principal es soportar y proteger el diente y unirlo al tejido óseo de los maxilares, además de proteger y mantener la integridad en la superficie de la mucosa masticatoria. Se divide en: 1) periodonto de protección, integrado por la encía, y 2) periodonto de soporte, este último constituido por el ligamento periodontal, el cemento radicular y el hueso alveolar. Ambos periodontos constituyen una unidad biológica y funcional que experimenta cambios con la edad y que también está sometida a modificaciones en su morfología, relacionadas con alteraciones funcionales y del ambiente bucal [1]. Durante la odontogénesis, proceso de formación y desarrollo de los dientes, también se da la formación de los tejidos periodontales [1].

La encía es la parte de la mucosa masticatoria que va desde la línea mucogingival, cubre las apófisis alveolares y llega hasta rodear la porción cervical de los dientes, insertándose en ellos; adquiere su forma y textura con la erupción de los dientes [2].

Anatómicamente, la encía se divide en marginal o no insertada, insertada e interdental. La encía marginal es el borde de la encía que rodea los dientes; se extiende desde la encía insertada adyacente al surco gingival hasta el margen de la encía. Suele tener $1 \mathrm{~mm}$ de ancho y forma la pared de tejido blando del surco gingival. Este surco, en forma de $\mathrm{V}$, es un espacio poco profundo alrededor del diente; su profundidad se determina clínicamente con la sonda periodontal. Cuando termina la erupción dental, en una encía clínicamente saludable, la profundidad sondeable es de 2 a $3 \mathrm{~mm}$, y representa la cantidad de encía libre. Esta medida es un parámetro diagnóstico importante de salud periodontal [1].

La encía insertada es la continuación de la encía marginal; es firme, resistente y está unida firmemente al periostio del hueso alveolar. En la superficie vestibular se extiende hasta la mucosa alveolar laxa y móvil, delimitando la unión mucogingival. El ancho de la encía insertada es otro parámetro clínico importante que se determina desde la proyección externa del fondo del surco hasta la unión mucogingival; varía en diferentes partes de la boca: generalmente es mayor en la región de los incisivos y menor en los segmentos posteriores. La variación puede oscilar entre 1 y $9 \mathrm{~mm}$ [1].
La encía interdental se ubica en el espacio interproximal por debajo del punto de contacto dental y se adapta a la forma de este. Su forma depende del punto de contacto entre los dientes vecinos y de la presencia o no de recesión gingival [1].

Dependiendo de ciertas características de la encía - como espesor, color, presencia de eminencias radiculares, forma y posición de la corona clínica $[2,3]$ - , se ha sugerido la presencia de tres biotipos periodontales: uno delgado, con papilas altas e incisivos alargados; otro grueso con papilas cortas e incisivos ligeramente cuadrados, y un tercer tipo que reúne características de los dos anteriores $[2,4]$

Uno de los objetivos más importantes de un tratamiento de maloclusiones es lograr una sonrisa armoniosa, incluyendo la interacción de los dientes, los tejidos de soporte y los tejidos extraorales del paciente [5]. Se han descrito dos tipos de sonrisa: la social, que es espontánea, asociada con alguna emoción de alegría y que no es repetible ni reproducible; y la posada, que es realizada por el paciente de manera consciente, es reproducible y es la que se usa comúnmente en las investigaciones [6]. Por otra parte, Tjan y Miller [7] dividieron la sonrisa en tres categorías: la sonrisa baja, que muestra menos del 75\% de la corona clínica de los dientes anteriores; la sonrisa promedio, que exhibe del 75 al $100 \%$, y la sonrisa alta, que muestra una banda ancha de encía, superior a la corona clínica de los dientes anteriores maxilares.

La sonrisa gingival fue definida por Peck et al. [8] como la exposición, en máxima sonrisa, de más de $2 \mathrm{~mm}$ de encía. La sonrisa gingival de más de $3 \mathrm{~mm}$ es uno de los hallazgos que afectan la percepción de estética de la sonrisa por parte de los pacientes y de los clínicos [9]. Este tipo de sonrisa, que se ha descrito como común en pacientes pediátricos, usualmente se corrige o disminuye la exposición de encía con la edad, aunque algunos pacientes adultos persisten con la exposición de encía exagerada al sonreír y presentan sonrisa gingival toda su vida [10].

La forma, el tamaño y la simetría de los tejidos periodontales, como parte del órgano alvéolo dentario, tienen una gran influencia en la percepción de la estética de la sonrisa. En el 2011, McLeod et al. [11] encontraron que el tamaño del corredor bucal, la desviación de la línea media, la exposición de encía al sonreír y la discrepancia gingival entre el incisivo central y el lateral fueron los aspectos 
más críticos al evaluar la sonrisa. Por su parte, en su estudio, Murakami et al. [5] encontraron que una exposición de encía promedio es lo que la población del común percibe como estética. Asimismo, Allen [12] y Kokich et al. [9], en sus estudios acerca de la atracción o no de la sonrisa, demostraron que los pacientes con características asimétricas en su encía y con líneas de sonrisa altas eran percibidos como "no tan estéticos". Kokich et al. [9] también reportaron que diferencias en la longitud de las coronas clínicas de los incisivos superiores (a partir de 1,5 a $2 \mathrm{~mm}$ ), son detectadas como antiestéticas ya que esto afecta los niveles de los cenits gingivales. Por su parte, en su estudio, Monaco et al. [13] hicieron una clasificación de la sonrisa gingival así: sonrisa gingival dentogingival, sonrisa gingival muscular, sonrisa gingival dentoalveolar y sonrisa gingival mixta. Los autores hacen énfasis en que no es difícil reconocer cuándo un paciente tiene una sonrisa gingival, lo difícil es identificar qué tipo específico de sonrisa tiene, y este es un aspecto fundamental a la hora de instaurar un tratamiento temprano que pueda evitar cirugías en la vida adulta.

En adultos y adolescentes se realizan diferentes tratamientos para eliminar o disminuir la sonrisa gingival, entre los que están: 1) corregir la falla en la erupción pasiva con cirugías periodontales de alargamiento coronal [14, 15]; 2) aplicar mecánicas intrusivas con minitornillos [16]; 3) inyectar sustancias como el Botox ${ }^{\circ}$ para disminuir la capacidad de elevación del labio superior [17], y 4) realizar una cirugía maxilofacial. En adolescentes, el tratamiento suele incluir mecánicas intrusivas ortodóncicas en combinación con cirugía periodontal [14]. Por el contrario, en niños, el tratamiento se realiza en los pacientes con casos severos de sonrisa gingival, intervenidos algunas veces mediante el control vertical del maxilar superior con tracciones extraorales [13]. Generalmente, los casos moderados y leves no se tratan y se espera autocorrección con el crecimiento del labio y con la erupción pasiva del periodonto [18].

Teniendo en cuenta que el periodonto es un aspecto fundamental en la estética de la sonrisa, que en la mayoría de protocolos de manejo de sonrisa gingival se aborda el aspecto periodontal, y que en la literatura científica se describen características del periodonto en población adulta, pero muy poco del periodonto de los pacientes pediátricos, y aún menos con sonrisa gingival, este estudio de casos y controles, tuvo como objetivo describir y evaluar las características del periodonto de un grupo de niños con sonrisa gingival y compararlo con las características del periodonto de un grupo de niños sin sonrisa gingival. Así mismo se evaluó la influencia de las características gingivales sobre la presencia de sonrisa gingival y sobre los diferentes tipos de maloclusión en niños en dentición mixta silente.

\section{Materiales y métodos}

Se realizó un estudio de casos y controles en Medellín, con un tamaño de muestra probabilístico, con una proporción de casos basada en la prevalencia de sonrisa gingival del $26 \%$ reportado por Peck et al. [8], con un poder del $80 \%$ y una significancia del 0,05 . La proporción de casos frente a controles fue de 1 a 3 y el tamaño de muestra calculado fue de 96 individuos ( 24 casos y 72 controles). Los criterios de inclusión fueron: niños en dentición mixta silente, sistémicamente sanos, con cualquier tipo de maloclusión, sin pérdidas prematuras de dientes, agenesias ni caries interproximales extensas; periodontalmente sanos y cuyos padres aceptaran participar en el estudio. Los criterios de exclusión fueron: pacientes que tuvieran o hubieran recibido algún tratamiento ortopédico $u$ ortodóncico o que tuvieran alguna alteración sindrómica que comprometiera el complejo cráneofacial, o con historia de un trauma severo en la zona dentoalveolar anterior.

La muestra se seleccionó entre estudiantes de tres instituciones educativas de Medellín, algunas de ellas con convenio vigente con la Facultad de Odontología de la Universidad de Antioquia, para la realización de las prácticas descentralizadas de sus estudiantes. Al final de la recolección de la muestra se obtuvieron 37 casos y 126 controles que cumplieron con los criterios de inclusión, es decir, $69 \%$ más de la muestra calculada, lo que garantizó una mayor potencia del estudio.

\section{Clasificación de los pacientes y examen clínico}

En un principio, se revisó a todos los estudiantes de los grados $2^{\circ}, 3^{\circ}, 4^{\circ}$ y $5^{\circ}$ de primaria de las escuelas seleccionadas, mediante una valoración clínica intraoral que permitió seleccionar a los niños que cumplían con todos los criterios de inclusión; a 
los niños seleccionados se les entregó el consentimiento informado para que sus padres o acudientes lo firmaran. Después, se realizó un examen clínico solo a los niños que entregaron a sus profesores el consentimiento firmado. En este examen, se incluyó la clasificación de las relaciones molares de acuerdo con la clasificación de Angle, las relaciones caninas, mediciones de overjet y overbite con una sonda periodontal calibrada, marcada con milímetros, tal y como lo reporta el DAI [19]. Además, y para clasificar al paciente en el grupo de sonrisa gingival (sG) o no sonrisa gingival (NSG), se registró clínicamente la cantidad de encía expuesta al sonreír y se hicieron las mediciones de las variables periodontales: cantidad de encía insertada, profundidad sondeable y biotipo periodontal. Estas mediciones periodontales se realizaron en cada diente anterior superior e inferior, siguiendo el protocolo de Botero y Bedoya [20], lo cual se hizo previa calibración de los evaluadores y habiendo seleccionado al evaluador que tuvo la concordancia mayor, con un kappa del 0,87.

\section{Toma de fotograma y análisis}

Después de tomar las medidas clínicas, y siguiendo el protocolo descrito por Ackerman et al. [6], se tomó un video frontal de la cara del paciente en sonrisa posada no forzada; de dicho video se escogió un fotograma 1:1 que representó la posición más reproducible de la sonrisa y se procedió a comparar la clasificación clínica del niño en el grupo de SG o en el de NSG con la del fotograma. Solo uno de los pacientes tuvo una clasificación diferente entre la valoración clínica y la del fotograma; en ese caso se priorizó la clasificación del fotograma.

\section{Control de sesgos}

El error aleatorio (de precisión) se controló calculando la muestra de manera probabilística, y el control del error sistemático (de validez) se hizo para seleccionar y medir. El control al sesgo de selección se realizó teniendo en cuenta los criterios de inclusión y exclusión. Para el sesgo de medición, se hizo un entrenamiento teórico de los evaluadores sobre los criterios dentales y periodontales a observar. Una vez se terminó el entrenamiento teórico, se evaluaron las variables a observar en 20 fotos clínicas de diversos pacientes y se compararon las diferencias hasta llegar a acuerdos en conjunto; en casos de desacuerdo, intervino un tercer evaluador. Después de este proceso, y para calibrar el instrumento de recolección de información y la medición de las variables, dos evaluadores las midieron en 11 pacientes diferentes a los de la muestra, utilizando una sonda periodontal calibrada marca Delta. Dicha observación se hizo cuatro veces, con un intervalo de dos semanas entre ellas, hasta lograr concordancia en las medidas intraoperador e interoperador (kappa 0,87 e ICC 0,96 ).

Luego se hizo una prueba piloto con 10 pacientes de la muestra, con el fin de ajustar el instrumento de recolección de la información y se determinó - según la concordancia obtenida por los evaluadores, y de común acuerdo entre ellos- que uno mediría las variables periodontales y otro las variables oclusales.

Para realizar el video, un experto en ayudas audiovisuales siguió el protocolo descrito por Ackerman et al. [6], tomando los videos en iguales condiciones de luminosidad y distancia del objeto. Asimismo, los evaluadores hicieron pruebas de calibración intraoperador e interoperador para seleccionar el fotograma que representara la posición de "sonrisa posada no forzada" y cualquier discrepancia fue acordada entre ellos con intervención de un tercero. La ubicación de puntos y la medición de las distancias en los fotogramas también se hicieron previa calibración del evaluador (ICC 0,96 ). Después se digitaron los datos en una tabla de Excel y se procedió a buscar datos atípicos o posibles errores de medición y a realizar el análisis estadístico.

\section{Aspectos éticos}

Como todos los pacientes son menores de edad, sus padres o acudientes firmaron el consentimiento informado, avalado por el Comité de Ética de la Facultad de Odontología de la Universidad de Antioquia, en el acta número 4 del 18 de junio del 2014. Esta investigación fue clasificada como de riesgo mínimo.

\section{Análisis estadístico}

Se utilizó el programa sPss versión 17 (sPss Inc, Chicago, III). Las variables cuantitativas se expresaron en promedios, más o menos desviaciones estándar y las variables nominales en frecuencias relativas y absolutas. Para estimar la asociación entre el tipo de sonrisa y la maloclusión, se hizo 
un análisis bivariado por medio del coeficiente de correlación de Pearson. Para determinar el comportamiento de las variables periodontales y de la maloclusión en los grupos de SG y NSG, se utilizó una t de Student. Por último, se hizo un análisis multivariado de regresión logística binaria para contrastar la influencia de las variables periodontales con la presencia o no de sonrisa gingival.

\section{Resultados}

Se evaluaron 1590 sujetos, de los cuales 163 cumplieron los criterios de inclusión y exclusión. Las características demográficas fueron similares en ambos grupos (tabla 1).

Según el tipo de maloclusión, se encontró que 104 pacientes tenían maloclusión clase I (63,8\%), 34 maloclusión clase II $(20,9 \%$ ) y 25 maloclusión clase III $(15,3 \%)$. La profundidad de surco encontrada en los pacientes fue de $1,7 \pm 0,3$. No se encontraron diferencias estadísticamente significativas en el comportamiento de las variables periodontales cuantitativas, de acuerdo con el tipo de sonrisa (tabla 2).

Con respecto a la profundidad sondeable y el tamaño de la encía insertada, y su relación con el tipo de maloclusión, el análisis no mostró diferencias (tabla 3).

El $76 \%$ de la muestra presentó un biotipo periodontal grueso y no se encontraron diferencias al comparar las maloclusiones con el biotipo periodontal (tabla 4).

Tabla 1. Distribución de la muestra según edad y sexo

\begin{tabular}{|l|l|l|l|l|}
\hline \multicolumn{2}{|c|}{ Variable } & \multicolumn{1}{|c|}{ Casos } & Controles & \multicolumn{1}{|c|}{ Valor p } \\
\hline \multirow{2}{*}{ Edad } & $8,8 \pm 0,9$ & $8,8 \pm 0,8$ & $0,836^{*}$ \\
\hline \multirow{2}{*}{ Sexo } & Hombre & $21(56,7 \%)$ & $76(60,3 \%)$ & \multirow{2}{*}{$0,698^{* *}$} \\
\cline { 2 - 4 } & Mujer & $16(43,3 \%)$ & $50(39,7 \%)$ & \\
\hline \multirow{2}{*}{ *Pruebat de Student ${ }^{* *}$ Prueba chi-cudrad de Pearson }
\end{tabular}

${ }^{\star}$ Prueba t de Student ${ }^{* \star}$ Prueba chi-cuadrado de Pearson.

Fuente: elaboración propia

Tabla 2. Variables periodontales cuantitativas según tipo de sonrisa

\begin{tabular}{|c|c|c|c|c|c|}
\hline Variable & Tipo de sonrisa & $\overline{\mathrm{X}}_{ \pm \mathbf{D E}}$ & Mínimo y máximo & IC $95 \%$ para el $\bar{X}$ & Valor $\mathrm{p} t \mathrm{de}$ Student \\
\hline \multirow{2}{*}{$\begin{array}{l}\text { Profundidad } \\
\text { sondeable }\end{array}$} & SG & $1,7 \pm 0,3$ & $1,0-2,3$ & $1,6-1,8$ & \multirow{2}{*}{0,689} \\
\hline & SNG & $1,7 \pm 0,3$ & $1,0-2,5$ & $1,6-1,7$ & \\
\hline \multirow{2}{*}{ Encía insertada } & SG & $4,0 \pm 0,7$ & $3,0-6,0$ & $3,8-4,3$ & \multirow{2}{*}{0,695} \\
\hline & SNG & $3,8 \pm 0,6$ & $2,5-5,7$ & $3,7-4,0$ & \\
\hline
\end{tabular}

Fuente: elaboración propia

Tabla 3. Relación variables periodontales y tipo de maloclusión

\begin{tabular}{|l|c|c|c|c|c|}
\hline Variable periodontal & Tipo maloclusión & $\overline{\mathbf{X}} \pm \mathbf{D E}$ & Mínimo y máximo & IC 95\% para el $\overline{\mathbf{X}}$ & Valor $\mathbf{p}$ t de Student \\
\hline Profundidad sondeable & I & $1,7 \pm 0,3$ & $1,0-2,5$ & $1,6-1,7$ & \\
\cline { 2 - 5 } & II & $1,7 \pm 0,3$ & $1,0-2,2$ & $1,5-1,8$ & \multirow{2}{*}{0,586} \\
\cline { 2 - 5 } & III & $1,7 \pm 0,2$ & $1,3-2,3$ & $1,6-1,8$ & \multirow{2}{*}{0,937} \\
\hline Encía insertada & I & $3,9 \pm 0,6$ & $2,5-6,0$ & $3,7-4,0$ & $3,7-4,1$ \\
\cline { 2 - 5 } & II & $3,9 \pm 0,5$ & $3,0-5,3$ & $3,6-4,2$ & \\
\cline { 2 - 5 } & III & $3,8 \pm 0,7$ & $3,0-5,3$ & & \\
\hline
\end{tabular}

Fuente: elaboración propia

$\mathrm{Al}$ analizar la influencia del biotipo periodontal (grueso o delgado) sobre la sonrisa gingival, se encontró un Odds Ratio (OR) de 1,6, lo cual sugiere que es un factor de riesgo, pero el intervalo de confianza mostró que este valor no fue estadísticamente significativo (tabla 5). 
Tabla 4. Distribución del biotipo periodontal según la maloclusión

\begin{tabular}{|c|c|c|c|c|c|}
\hline & \multicolumn{3}{|c|}{ Tipo de maloclusión } & \multirow{2}{*}{ Total } \\
\hline & & I & II & III & \\
\hline \multirow{2}{*}{ Biotipo } & $\begin{array}{c}\text { Grueso } \\
(\mathrm{n} \%)\end{array}$ & $75(60,5)$ & $30(24,2)$ & $19(15,3)$ & $124(76,1)$ \\
\hline & $\begin{array}{c}\text { Delgado } \\
(\mathrm{n} \%)\end{array}$ & $29(74,4)$ & $4(10,2)$ & $6(15,4)$ & $39(23,9)$ \\
\hline
\end{tabular}

Fuente: elaboración propia

Tabla 5. Influencia del biotipo periodontal sobre la sonrisa gingival

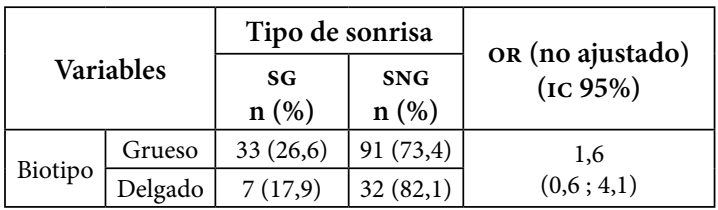

Fuente: elaboración propia

\section{Discusión}

La sonrisa es un aspecto esencial al evaluar el resultado de un tratamiento de ortodoncia [9]; una exposición alta de encía al sonreír es un factor percibido como "no estético" por parte tanto del clínico, como del paciente $[12,21]$. La exposición aumentada de encía en niños es percibida como normal [22], pero en adultos es catalogada como antiestética $[9,12$, 21]. Este estudio, con un diseño de casos y controles, buscó indagar en la etiología de la sonrisa gingival evaluando las características periodontales en niños con sonrisa gingival y comparándolas con las de un grupo de niños sin sonrisa gingival.

El estadio de desarrollo de la oclusión es un factor importante en los niños, ya que la oclusión es un proceso dinámico que sufre muchos cambios durante el crecimiento. Se ha encontrado que la edad cronológica no es un buen factor para evaluar el desarrollo oclusal, y por eso los clínicos prefieren usar la edad dental para clasificar a los pacientes [23]. Por esta razón, en este estudio, se decidió evaluar a los pacientes en dentición mixta silente, que es el momento de la oclusión en que menos cambios se producen desde el punto de vista clínico [24].

No se encontraron diferencias entre la edad y el sexo de los pacientes con y sin sonrisa gingival. Esto y el cumplimiento estricto de los criterios de inclusión y exclusión implican que los grupos fueron similares y, por lo tanto, comparables. La edad promedio de los pacientes fue de $8,8 \pm 0,9$ años para los casos, y de $\pm 0,8$ para los controles; $y$ aunque hubo mayor proporción de niños que de niñas en ambos grupos, esta diferencia no fue estadísticamente significativa.

En relación con el sexo, algunos autores han mencionado ciertas diferencias entre la encía de hombres y mujeres $[2,8]$. El presente estudio no las encontró, lo cual sugiere que el dimorfismo sexual no se ha expresado en edades tempranas y, por lo tanto, no se vieron diferencias entre los sexos.

La maloclusión que más se presentó en la población estudiada fue la clase I $(63,8 \%)$. Diferentes autores han encontrado un patrón similar en diversas poblaciones, así: Kirzioglu et al. [25] encontraron que un $73,6 \%$ de la población turca evaluada presentaba una relación molar de clase I, lo que indica que este grupo fue más prevalente; sin embargo, en su estudio Emine, también en población turca, encontró mayor prevalencia de clase II que de clase i [23]. En México, en población aborigen, se encontró mayor porcentaje de maloclusión clase II $(46,33 \%)$, seguido de la clase III $(38,98 \%)$ y, por último, la clase i $(13,56 \%)$ [26]. En población albanesa, también se encontró mayor prevalencia de clase I $(40 \%)$ seguida de la clase II, $(29,8 \%)$ y la maloclusión menos presente fue la clase III [27]. Así mismo, los estudios en diferentes poblaciones muestran diferentes patrones. Parece ser que la raza, las características demográficas de la población y la selección de la muestra tienen implicaciones importantes en ese sentido.

La profundidad del surco gingival es un factor que permite calcular si al diente le falta erupción pasiva y si es posible realizar un procedimiento quirúrgico de alargamiento coronal. En pacientes jóvenes, dicha profundidad debe tener valores de entre 1 y $3 \mathrm{~mm}$ y en adultos, por lo general, es de 1 $\mathrm{mm}$ [1]. Con respecto a la profundidad del surco, en este estudio se encontró que la medida de profundidad sondeable no fue diferente entre el grupo de pacientes con sonrisa gingival y el grupo sin sonrisa gingival. Esto implica que en pacientes con profundidad del surco normal esta variable no es la que hace la diferencia en la presencia de la sonrisa gingival, como sí podría ser en pacientes que tengan problemas con la erupción pasiva $[12,14]$.

El tamaño de la encía insertada tampoco mostró diferencias entre los dos grupos. Este es un factor clave, sobre todo en pacientes con excesos verticales 
y maloclusión de clase ir [28] que han mostrado una alta prevalencia de sonrisa gingival. Esto quiere decir que, aunque la sonrisa gingival podría estar relacionada con exceso vertical del maxilar superior [8], el tamaño de la encía insertada no parece estar relacionado.

Con respecto a la relación entre las características periodontales y las maloclusiones, este estudio no encontró ningún patrón específico, ni ninguna relación entre estos dos. Algunas investigaciones sugieren una relación entre la maloclusión clase III y el aumento en la recesión del hueso alveolar y disminución en la amplitud de la encía insertada, pero más relacionado con el tratamiento prequirúrgico de la clase inI y la proinclinación de los incisivos inferiores, que con el biotipo específico en sí [29]. En su estudio del 2012, Sawawi et al. [30] evaluaron 200 pacientes y tampoco encontraron relación alguna entre la maloclusión y el biotipo periodontal. Esto sugiere entonces que no es la maloclusión de base la que juega un factor determinante en las variables periodontales, sino que es más importante la posición específica de los dientes sobre las bases óseas. Asimismo, estos autores invitan al clínico a evaluar muy bien la posición y la proinclinación que le hará a los dientes para evitar defectos periodontales futuros.

Lo que sí se ha encontrado es que existen diferencias en el biotipo periodontal al relacionarlo con el sexo. En el 2014, Sawawi y Al-Zahrani [31] encontraron que las mujeres tienen 4,2 veces más riesgo de tener biotipo periodontal delgado en los incisivos superiores y 5 veces más en los incisivos inferiores que los hombres.

En el 2006, Szarmach et al. [32] encontraron que los factores anatómicos, la maloclusión y las irregularidades dentales fueron la causa de recesiones únicas o múltiples en pacientes en tratamiento ortodóncico. Asimismo, encontraron diferencias estadísticamente significativas entre el biotipo periodontal y la recesión gingival, indicando que pacientes con biotipo delgado son más propensos a ella. De manera similar a lo hallado en la presente investigación, dichos autores tampoco encontraron una relación específica entre maloclusiones y alteraciones periodontales.

Por último, al analizar la influencia de las variables periodontales sobre la presencia o no de sonrisa gingival, este estudio encontró un odds ratio de 1,6 para sonrisa gingival con el biotipo periodontal grueso. Sin embargo, esta diferencia no fue estadísticamente significativa (tabla 5). En el 2011, Cook et al. [33] encontraron una asociación estadísticamente significativa entre el biotipo periodontal grueso y el espesor de la lámina ósea vestibular, lo que sugiere una relación entre la forma de la encía y la forma del hueso subyacente. Por otro lado, en su estudio, Polack y Mahn [34] mencionan que la exposición gingival al sonreír se relaciona con coronas clínicas cortas y hueso vestibular grueso. Esto sugiere, de manera indirecta, que los pacientes con sonrisa gingival podrían tener alguna asociación con biotipo periodontal grueso. Esta asociación no fue confirmada en la presente investigación, y debería ser objeto de análisis futuros.

\section{Conclusiones}

Aunque los aspectos periodontales son determinantes en la estética de la sonrisa y son importantes a la hora de planear el tratamiento en adultos, en niños en dentición mixta silente no se encontró influencia de los factores periodontales (profundidad sondeable, amplitud de encía insertada y biotipo periodontal), sobre la presencia o no de sonrisa gingival. Sin embargo, y de acuerdo con otro autores [33] [34], parece ser que el biotipo periodontal grueso puede estar asociado con sonrisa gingival. Este aspecto requerirá más investigación.

\section{Agradecimientos}

Los autores agradecemos al doctor Luis Gonzalo Álvarez por sus valiosos aportes en el manejo de los datos y en el análisis de la información, y al doctor Javier Botero por su colaboración en la calibración de las variables periodontales.

\section{Referencias}

[1] Newman M, Takei HH, Klokkevold PR. La encía: estructuras de soporte dentario. En: Newman MG, editor. Periodontología clínica. 10a. ed. México: McGraw-Hill; 2006. p. 46-68. 
[2] Alarcón-Palacios MA, Lister-Blondet CR. Fenotipos periodontales. Rev Estomatol Hered. 2010;20(4):227-30.

[3] Müller HP, Heinecke A, Schaller N, Eger T. Masticatory Mucosa in Subjects with Different Periodontal Phenotypes. J Clin Periodontol. 2000;27(9):621-6.

[4] De Rouck T, Eghbali R, Collys K, De Bruyn H, Cosyn J. The gingival biotype revisited: transparency of the periodontal probe through the gingival margin as a method to discriminate thin from thick gingiva. J Clin Periodontol. 2009;36(5):428-33. doi: 10.1111/j.1600-051X.2009.01398.x.

[5] Murakami Y, Deguchi T, Kageyama T, Miyazawa $\mathrm{H}$, Foong KWC. Assessment of the esthetic smile in young Japanese women. Orthod Waves. 2008;67(3):104-12. doi: 10.1016/j.odw.2008.03.001.

[6] Ackerman JL, Ackerman MB, Brensinger CM. A morphometric analysis of the posed smile. Clin Orthod Res. 1998;1(1):2-11.

[7] Tian A, Miller Gary TJ. Some esthetic factors in a smile. J Prosthet Dent. 1984;51(1):24-8.

[8] Peck S, Peck L, Kataja M. The Gingival Smile Line. Angle Orthod. 1992;62 (2):91-100.

[9] Kokich VO, Kokich VG, Kiyak HA. Perceptions of dental professionals and laypersons to altered dental esthetics: Asymmetric and symmetric situations. Am J Orthod Dentofac. Orthop 2006;130(2):141-51. doi: 10.1016/j.ajodo.2006.04.017.

[10] Ong M, Wang H-L. Periodontic and orthodontic treatment in adults. Am J Orthod Dentofac. Orthop 2002;122(4):420-428. .

[11] McLeod C, Fields HW, Hechter F, Wiltshire W, Rody W, Christensen J. Esthetics and smile characteristics evaluated by laypersons. Angle Orthod. 2011;81(2):198-205. doi: 10.2319/060510-309.1.

[12] Allen E. Use of mucogingival surgical procedures to enhance esthetics. Dent Clin North Am. 1988;32(2):307-30.

[13] Monaco A, Streni O, Marci MC, Marzo G, Gatto R, Giannoni M. Gummy smile: clinical parameters useful for diagnosis and therapeutic approach. J Clin Pediatr Dent 2004;29(1):19-25.

[14] Mele M, Stefanini M, Marzadori M, Mazzotti Zucchelli G. Gummy smile. Periodontal treatment in patients with passive altered eruption. J Parodontol d'Implantologie Orale 2010;29(4):287.

[15] Cairo F, Graziani F, Franchi L, Defraia E, Pini Prato GP. Periodontal plastic surgery to improve aesthetics in patients with altered passive eruption/gummy smile: a case series study. Int J Dent. 2012;ID 837658. doi:10.1155/2012/837658.

[16] Updhayay M, Nagaray K, Yadav S. Mini-implants for en masse Intrusion of maxillary anterior teeth in a severe class II division 2 malocclusion. J Orthod. 2008;35(2):79-89.

[17] Singh H, Srivastava D, Sharma P, Kapoor P, Roy P. Redefining treatment of gummy smile with Botox-a report of three cases. Int J Orthod Milwaukee Wis. 2014;25(4):63-6.

[18] Sarver DM. Growth maturation aging: how the dental team enhances facial and dental esthetics for a lifetime. Compend Contin Educ Dent. 2010;31 (4):274-80, 282-3; quiz 284, 287.

[19] Pérez VA, García G, Cárdenas A, Carrasco R, Castro C, Lezama G. et al. Índice Estética Dental (DAI) y necesidad de tratamiento ortodóncico en escolares, Verano 2007. Oral. 2008;9:472-5.

[20] Botero JE, Bedoya E. Determinantes del diagnóstico periodontal. Rev Clin Periodoncia Implant Rehabil Oral. 2010;3(2):94-9.

[21] Kokich VO, Kiyak A, Shapiro PA. Comparing the perception of dentists and lay people to altered dental esthetics. J Esthet Dent. 1999;11:311-24.

[22] Bernal LV, Zapata Ó, Tobón C, Gómez MI, Suárez J. Características de la sonrisa en pacientes infantiles con normoclusión. Rev Fac Odontol Univ Antioq. 2015;27(1):11-29. doi: http://dx.doi.org/10.17533/ udea.rfo.v27n1a1

[23] Emine K, Lale T, Kahraman G. Distribution of sagittal occlusal relationships in different stages of dentition. Braz Oral Res. 2015;29(1):1-6. doi:10.1590/1807-3107BOR-2015.

[24] Ocampo P, Parra N, Botero P. Guía de erupción y extraccion seriada. Una mirada desde el desarrollo de oclusión. Rev Nac Odontol. 2013;9(edición especial):25-35.

[25] Kirzioglu Z, Simsek S, Yilmaz Y. Longitudinal occlusal changes during the primary dentition and during the passage from primary dentition to mixed dentition among a group of Turkish children. Eur Arch Paediatr Dent. 2013;14(2):97-103.

[26] Aamodt K, Reyna-Blanco O, Sosa R, Hsieh R, De la Garza Ramos M, García Martínez M, et al. Prevalence of caries and malocclusion in an indigenous population in Chiapas, Mexico. Int Dent J. 2015;65(5):249-55. doi:10.1111/idj.12177.

[27] Laganà G, Masucci C, Fabi F, Bollero P, Cozza P. Prevalence of malocclusions, oral habits and orthodontic treatment need in a 7 - to 15 -year-old schoolchildren population in Tirana. Prog Orthod. 2013;14(1):1-7. doi: 10.1186/2196-1042-14-12.

[28] Wu H, Lin J, Zhou L, Bai D. Classification and craniofacial features of gummy smile in adolescents. J Craniofac Surg. 2010;21(5):1474-9. doi: 10.1097/ scs.0b013e3181edc627. 
[29] Choi YJ, Chung CJ, Kim K-H. Periodontal consequences of mandibular incisor proclination during presurgical orthodontic treatment in Class III malocclusion patients. Angle Orthod. 2015;85(3):42733. doi: 10.2319/021414-110.1.

[30] Zawawi K, Al-Harthi S, Al-Zahrani M. Prevalence of gingival biotype and its relationship to dental malocclusion. Saudi Med J. 2012;33(6):671-5.

[31] Zawawi KH, Al-Zahrani MS. Gingival biotype in relation to incisors' inclination and position. Saudi Med J. 2014;35(11):1378-83.
[32] Szarmach I, Wawrzyn-Sobczak K, Kaczyńska J, Kozłowska M, Stokowska W. Recession occurrence in patients treated with fixed appliances-preliminary report. Adv Med Sci. 2006;51:213-6.

[33] Cook D, Mealey B, Verrett R, Mills M, Noujeim M, Lasho D et al. Relationship between clinical periodontal biotype and labial plate thickness: an in vivo study. Int J Periodontics Restor Dent. 2011;31(4):345-54.

[34] Polack M, Mahn D. Biotype change for the esthetic rehabilitation of the smile. J Esthet Restor Dent. 2013;25(3):177-86. doi: 10.1111/jerd.12029. 